\title{
COMPLEXIFYING LIE GROUP ACTIONS ON HOMOGENEOUS MANIFOLDS OF NON-COMPACT DIMENSION TWO
}

\author{
S. RUHALLAH AHMADI AND BRUCE GILLIGAN
}

\begin{abstract}
If $X$ is a connected complex manifold with $d_{X}=2$ that admits a (connected) Lie group $G$ acting transitively as a group of holomorphic transformations, then the action extends to an action of the complexification $\widehat{G}$ of $G$ on $X$ except when either the unit disk in the complex plane or a strictly pseudoconcave homogeneous complex manifold is the base or fiber of some homogeneous fibration of $X$.
\end{abstract}

\section{INTRODUCTION}

A useful invariant for non-compact manifolds in the setting of proper actions of Lie groups is the notion of non-compact dimension that was introduced by Abels in [Abe76]; see also $\S 2$ in [Abe82]. We take the dual, but equivalent, approach and do not assume that any Lie group action is necessarily proper. For $X$ a connected (real) smooth manifold we define $d_{X}$ to be the codimension of the top non-vanishing homology group of $X$ with coefficients in $\mathbb{Z}_{2}$. The invariant $d_{X}$ measures, in a certain sense, how far the manifold $X$ is from being compact and this invariant is applied here to study a particular case of the following problem.

Suppose a Lie group $G$ is acting as a group of holomorphic automorphisms on a complex space $X$. One can ask whether the complexification $\widehat{G}$ of $G$ (in the sense of Chapter XVII.5 in Hoch65) acts holomorphically on a complex space $\widehat{X}$ into which $X$ can be $G$-equivariantly embedded as an open subset. A special case of this problem is to attempt to choose $\widehat{X}=X$. Certainly, the automorphism group of a compact complex manifold is a complex Lie group BoMo47] and in the compact case the action always extends to an action of the complexification of the group involved on the compact complex space. But such an extension does not exist in all settings, e.g., if $X$ is hyperbolic. However, it was proved in Theorem 3.1 in [GH98] that if $X$ is a complex manifold with $d_{X}$ equal to one that is homogeneous under the action of a Lie group $G$ acting by holomorphic transformations, then there is a corresponding transitive $\widehat{G}$-action on $X$.

In this paper we consider complex manifolds that have a transitive action of a real Lie group acting as a group of holomorphic transformations and that satisfy the condition that $d$ is equal to two. We wish to resolve the problem stated above in this setting, i.e., determine when one can extend the given action of a $G$ on such a

1991 Mathematics Subject Classification. Primary 32M10.

Key words and phrases. homogeneous complex manifold, non-compact dimension two, complexification.

This work was partially supported by an NSERC Discovery Grant for which the authors are grateful. We also thank Prof. A. T. Huckleberry and the referee for their comments that led to improvements in the work. 
complex manifold $X$ to the holomorphic action of its complexification $\widehat{G}$ on $X$. Of course, this is not always possible in the setting of $d=2$, as obvious examples are the unit disk in the complex plane, or more generally, the complement of a closed ball in higher dimensional projective space which fibers real analytically as a disk bundle over a compact manifold.

It turns out that there is only one other type of example where the extension problem has a negative answer arising in the following way. Let $B=K / L$ be a compact rank one symmetric space and consider its tangent bundle $T(B)$ together with its natural Stein manifold structure and induced $K$-action, see Proposition 2 in MN63. Let $Y$ be a $K$-equivariant compactification of $T(B)$ to a flag manifold obtained by adding an ample divisor. Now set $X:=Y \backslash B$. Heuristically one can view this setting in the following way. Since $T(B)$ is Stein, it admits a strictly pseudoconvex exhaustion, which when observed from the other side endows $X$ with the structure of a strictly pseudoconcave homogeneous manifold. Such manifolds were classified in [HS81. Of course, the extension problem also fails for homogeneous manifolds where such examples occur as fiber or base in any equivariant fibration of the manifolds.

The purpose of this note is to prove the following.

Theorem 1.1. Suppose $G$ is a connected Lie group acting transitively and almost effectively as a group of holomorphic transformations on a complex manifold $X=G / H$ with $d_{X}=2$. Then the complexification $\widehat{G}$ of $G$ acts holomorphically and transitively on $X$ unless the unit disk in the complex plane or a strictly pseudoconcave homogeneous manifold is either the fiber or base of some homogeneous fibration of $X$.

The methods that we use are now classical, e.g., see HO84, OR84, GH98, and Gil95. In particular, we use some basic fibrations from loc. cit. along with a Fibration Lemma from AG94 for dealing with the invariant $d$ in the setting of fibrations. And the paper is organized as follows. In section two we discuss the exceptional flag domains that can occur. In section three we present a technical Lemma needed later. Finally, the proof of the Theorem is given in section four.

\section{The Exceptional Flag Domains}

Suppose $\widehat{G}$ is a connected complex semisimple Lie group, $\widehat{P}$ is a parabolic subgroup of $\widehat{G}$ and let $Y:=\widehat{G} / \widehat{P}$ be the corresponding flag manifold. Any real form $G$ of $\widehat{G}$ has a finite number of orbits in $Y$ and so at least one orbit must be open. Such an open orbit is called a flag domain. For detailed information about this setting we refer the interested reader to Wol69] and [FHW]. We only recall here that the group $\operatorname{Aut}(Y)$ can be faithfully represented into $\operatorname{Aut}\left(\mathbb{P}_{N}\right)$ for some equivariant embedding of $Y$ into a projective space $\mathbb{P}_{N}$ and so we are dealing with linear groups and are in an algebraic setting throughout.

Let $K$ be a maximal compact subgroup of $G$ and $K^{\mathbb{C}}$ be its complexification in $\operatorname{Aut}(Y)$. Matsuki duality (see Mat79] and Mat82; for a geometric approach see Theorem 8.3.1 in [FHW] states that to every $G$-orbit in the flag manifold $Y$ there is a unique $K^{\mathbb{C}}$-orbit which intersects the $G$-orbit in a $K$-orbit and vice versa. If the $G$-orbit is open (resp. closed), then its dual $K^{\mathbb{C}}$-orbit is closed (resp. open). 
Since there is a unique closed $G$-orbit $A$ in $Y$ which is also a $K$-orbit (Theorem 3.5 and Corollary 3.4, Wol69), it follows from Matsuki duality that there is a unique open $K^{\mathbb{C}}$-orbit $W$ in $Y$ which contains $A$. Since the $K^{\mathbb{C}}$-action is algebraic, the isotropy subgroup for this action is algebraic and thus has a finite number of connected components. As a consequence $W$ has either one or two ends (see Bor53] when the isotropy group is connected and Proposition 1 Gil91 when the isotropy group has a finite number of connected components).

We are interested in the special case of a flag domain $X$ with $d_{X}=2$. Our approach is to study the $K$-orbits in the manifold $X$. Now the unique complex $K$-orbit $E$ in the flag domain $X$ is the unique orbit of minimal dimension and is a strong deformation retract of $X$, e.g., see Wol69]. We now use this fact.

Proposition 2.1. Let $X$ be a flag domain homogeneous under a real form $G$ of a complex semisimple Lie group $\widehat{G}$ acting as a group of holomorphic transformations and assume $d_{X}=2$. Then $X$ is a non-compact strictly pseudoconcave homogeneous manifold. In particular, $\widehat{G}$ does not act transitively on $X$.

Proof. As we noted above, $W$ has either one or two ends and we first suppose that $W=K^{\mathbb{C}} / L^{\mathbb{C}}$ has two ends, where $L^{\mathbb{C}}$ denotes the isotropy subgroup. Since we are in an algebraic setting, there exists a parabolic subgroup $\widehat{P}$ of $K^{\mathbb{C}}$ and a character $\chi: \widehat{P} \rightarrow \mathbb{C}^{*}$ such that $L^{\mathbb{C}}=$ ker $\chi$, see Akh77. In particular, $W$ fibers as a $\mathbb{C}^{*}$-bundle over the flag manifold $Q:=K^{\mathbb{C}} / \widehat{P}$. Since any $K$-orbit in $W$ is mapped surjectively onto the base $Q$, all $K$-orbits in $W$ have real codimension at most two. Now suppose there would be a $K$-orbit $B$ in $W$ that has real codimension two. We claim that this assumption yields a contradiction. Since $Q$ is simply connected, $B$ is a topological section of the $\mathbb{C}^{*}$-principal bundle $W$ and thus $W$ is topologically trivial. But $H^{1}(Q, \mathcal{O})=0$, because $Q$ is a flag manifold. So the line bundle associated to $W$ by adding a 0 -section is analytically trivial. Thus $W$ as a bundle is also analytically trivial. This means that all $K$-orbits in $W$ are complex hypersurfaces, contradicting the fact that in the flag domain $X$ there is a unique $K$-orbit which is complex, see Lemma 5.1 in Wol69. As a result all $K$-orbits in $W$ have real codimension one, i.e., are real hypersurfaces.

We are now in the following setting. The group $K^{\mathbb{C}}$ has one open orbit in $Y$ and two that are closed. By Matsuki duality the group $G$ correspondingly has two open orbits and one closed orbit with the latter being exactly a real hypersurface $K$-orbit in $W$. This setting is described in Theorem 5.2 in GH09, where it is

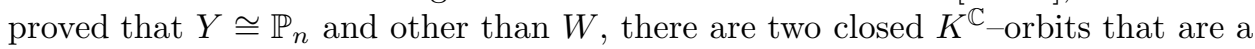
$\mathbb{P}_{q-1}$ and a $\mathbb{P}_{p-1}$ with $(q-1)+(p-1)=n-1$. Since $X=W \cup \mathbb{P}_{q-1}$ and $d_{X}=2$, it follows that $\mathbb{P}_{q-1}$ is a complex hypersurface in $X$, i.e., $q-1=n-1$. From the above equality we see that $p=1$ and so $\mathbb{P}_{p-1}$ is a point. The groups that can occur in this setting are also described in Theorem 5.2 in [GH09.

Now suppose $W$ has one end. By the discussion given before the statement of the Proposition, the flag manifold $Y$ consists of two $K^{\mathbb{C}}$-orbits, namely, $W$ and $E$, where $W$ is the open orbit and $E$ is a complex hypersurface orbit. Since we are in the setting of algebraic group actions on a flag manifold, this set up was analyzed in Akh79 using classification results of Wang Wan52. The open $K^{\mathbb{C}}$-orbit is the tangent bundle $T(B)$ of a compact rank one symmetric space $B$. 
Remark 2.2. We refer the reader to [HS81] for a very detailed description of these non-compact strictly pseudoconcave homogeneous manifolds. It follows from the classification given there that $X$ is the complement of the closure of a ball in $\mathbb{P}_{n}$ which is the flag manifold $Y$ containing $X$ or $X=Y \backslash B$ with $Y$ the compactification of the tangent bundle $T(B)$ of a compact rank one symmetric space $B$. The list of these spaces is as follows: the $n$-sphere, $\mathbb{S}^{n}, n \geq 2$, real projective space, $\mathbb{R P}_{n}$, $n \geq 2$, complex projective space $\mathbb{P}_{n}, n \geq 1$, quaternionic projective space $\mathbb{Q P}_{n}$, $n \geq 1$, and the Cayley projective plane $F_{4} / \operatorname{Spin}(9)$.

\section{A teChNicAl Lemma}

We will need the following technical result in the proof of the theorem.

Lemma 3.1. Let $Y=\widehat{G} / \widehat{H}$ be an orbit of a connected complex semisimple Lie group $\widehat{G}$ acting holomorphically on some projective space and assume that a real form $G$ of $\widehat{G}$ has an open orbit $X:=G / H$ in $Y$ with $d_{X}=2$. Then $d_{Y} \leq d_{X}$.

Proof. If $Y$ is compact, then we are done because $d_{Y}=0$. So we assume throughout the rest of the proof that $Y$ is not compact. Let $K$ be a maximal compact subgroup of $G$. As we are in the setting of algebraic groups, there is a Mostow fibration, see Mos55, of the homogeneous manifold $X$ as a real vector bundle over a minimal $K$-orbit $M$. By assumption $d_{X}=2$ and thus $M$ has real codimension two. So the generic $K$-orbits in $X$ have real codimension one or two.

Suppose these orbits all have codimension two. If these orbits are complex, then they are also $K^{\mathbb{C}}-$ orbits, i.e., $Y$ is fibered by flag manifolds. One then has a fibration

$$
Y=\widehat{G} / \widehat{H} \longrightarrow \widehat{G} / K^{\mathbb{C}} \cdot \widehat{H}=: B,
$$

where $B$ is one dimensional and non-compact. If $B$ is biholomorphic to $\mathbb{C}$, then $d_{Y}=d_{\mathbb{C}}=2$. If $B=\mathbb{C}^{*}$, then $X=Y$ and we are again done. In passing, note that $Y$ splits as a product. And, if the $K$-orbits are not complex, then by the classification result given in Theorem 5.6 in GH09] it follows that $Y$ is compact and we assumed above that this is not the case.

So we now assume that the generic $K$-orbits in $X$ are real hypersurfaces. In this setting the complexification $K^{\mathbb{C}}$ of $K$ has an open orbit $Z$ which is Zariski open in its Zariski closure $\bar{Z}$. We set $E:=\bar{Z} \backslash Z$ and note that $Z \subset Y$, since $K^{\mathbb{C}}$ is a subgroup of $\widehat{G}$ and $Y \neq \bar{Z}$, since $Y$ is not compact. We can equivariantly modify $\bar{Z}$ so that the exceptional set $E$ has pure codimension one Ka67 and is equivariantly desingularized Hir70. By Lemma 2.2 in HS82 the compact group $K$ acts transitively on the components of $E$. In particular, the "infinity" component $E_{\infty}$ of $E$ is a flag manifold that is an orbit of both $\widehat{G}$ and $K^{\mathbb{C}}$. Note that $\widehat{G} \neq K^{\mathbb{C}}$, since, otherwise, $G=K$ would be a compact real form and $X$ would be compact, contrary to our assumption that $d_{X}=2$.

We first consider the case where $\widehat{G}$ is simple and prove the general case later. There are only certain possibilities that can arise when two different complex simple groups act transitively on a flag manifold and the classification was given by Onishchik [Oni62]. As noted for example in the Main Theorem in [Ste82], the isotropy of the $\widehat{G}$-action on $E_{\infty}$ is a maximal parabolic subgroup of $\widehat{G}$. As a consequence there is no proper fibration of $E_{\infty}$. We now consider what this tells us about $Y$. 
If $Y$ is Stein, then $\widehat{H}$ is reductive, see [Mat60 and Oni60. Then $G$ acts transitively on $Y$ Oni69] and so $X=Y$. Otherwise, the Weisfeiler construction Weis66] yields a fibration $Y=\widehat{G} / \widehat{H} \rightarrow \widehat{G} / \widehat{I}$ with $\widehat{I}$ a parabolic group containing $\widehat{H}$ and $F:=\widehat{I} / \widehat{H}$ Stein. By virtue of the fact that there is no proper fibration of $E_{\infty}$ it follows that the fiber $F$ has complex dimension one. Note that $F$ is non-compact, since $Y$ is. Suppose first that $F=\mathbb{C}$ and so $Y$ is a $\mathbb{C}$-bundle over the flag manifold $Q:=\widehat{G} / \widehat{I}$. Since $Q$ is compact and simply connected, the Fibration Lemma AG94. implies $d_{Y}=2=d_{X}$. Otherwise, $F=\mathbb{C}^{*}$ and $Y$ is a $\mathbb{C}^{*}$-bundle over $Q$. But then the $G$-action on $F$ has an open orbit and this is only possible if this orbit is all of $F$. Thus $X=Y$ and this observation completes the proof when $\widehat{G}$ is simple.

We now assume that $\widehat{G}$ is semisimple. In general, if $G$ is simple, it is not necessarily the case that $\widehat{G}$ is simple. However, we claim that this is so in our setting. First note that $G$ is simple if and only if $K$ is simple if and only if $K^{\mathbb{C}}$ is simple. If $\widehat{G}$ is not simple, then $E_{\infty}$ is a product on which $K^{\mathbb{C}}$ acts transitively, e.g., see p. 1147 in Wol69. It follows that $K^{\mathbb{C}}$ is not simple loc. cit. and, as a consequence, we see that if $G$ is simple, then $\widehat{G}$ is simple. Now let $G=G_{1} \times G_{2}$ be a decomposition of $G$ with $G_{1}$ simple and let $K=K_{1} \times K_{2}$ be the corresponding decomposition of the maximal compact subgroup $K$ with $K_{i}$ a maximal compact subgroup of $G_{i}$ for $i=1,2$. We fiber the open $\widehat{G}$-orbit $Y$ by $\widehat{G}_{2}$ to get a fibration

$$
p: Y=\widehat{G} / \widehat{H} \stackrel{F}{\longrightarrow} \widehat{G} / \widehat{G}_{2} \cdot \widehat{H} .
$$

Since the generic $K$-orbits in $X$ are real hypersurfaces, there are two possibilities. The first case occurs when the generic $K_{1}$-orbit is a real hypersurface in $p(X)$ and $F$ is compact. Then the quotient $p(X)$ also has a divisor at infinity that is $\widehat{G}_{1}$-invariant, $G_{1}$ has an open orbit, and the generic $K_{1}$-orbits are hypersurfaces. In other words we are in the setting we considered above where we showed that either $p(X)=p(Y)$ or $d_{p(Y)}=2$. Since the fiber $F$ is compact, it follows that either $X=Y$ or $d_{Y}=2$. Otherwise, the generic $K_{2}-$ orbit in the $G_{2}-$ orbit is a real hypersurface and $p(X)=p(Y)$ is compact. Since the $G_{2}$-orbit in $F$ is open and has a divisor at infinity that is $\widehat{G}_{2}$-invariant, the result now follows by induction on the number of simple factors constituting the group $\widehat{G}$.

Remark 3.2. Suppose $X$ is an open $G$-orbit in $Y:=\widehat{G} / \widehat{H}$, where $\widehat{H}$ is a closed complex subgroup of the complex semisimple Lie group $\widehat{G}$. The question whether $d_{Y} \leq d_{X}$ holds in this setting without any additional assumptions on $d_{X}$ and $\widehat{H}$ is, as far as we know, open and there are no known counterexamples. In passing we note some cases where this question has an affirmative answer.

If $\widehat{H}$ is reductive algebraic, then $G$ acts transitively on $Y$ Oni69. The existence of such an open $G$-orbit in $\widehat{G} / \widehat{H}$ implies the existence of a proper complex subalgebra $\mathfrak{m}$ of the Lie algebra $\widehat{\mathfrak{g}}$ of $\widehat{G}$ such that one has a decomposition $\widehat{\mathfrak{g}}=\mathfrak{g}+\mathfrak{m}$ which is called a local factorization of $\widehat{G}$. These local factorizations have been studied in detail in certain cases; we refer the interested reader to Oni69, Mal77, Akh13, and the references cited there.

If $\widehat{H}$ is parabolic, then $X$ is a flag domain in the flag manifold $Y$ which is compact. In this case one has $0=d_{Y} \leq d_{X}$. Malyshev Mal75 proved that if a real form $G$ of inner type (he calls this first category) has an open orbit in $\widehat{G} / \widehat{H}$, 
then $\widehat{H}$ is parabolic. An example where $G$ has an open orbit in $\widehat{G} / \widehat{H}$ with $\widehat{H}$ not parabolic is given in Example 5.5 in Akh13 with $\widehat{\mathfrak{h}}$ an ideal in $\widehat{\mathfrak{p}}$, the Lie algebra of a parabolic subgroup $\widehat{P}$ that contains $\widehat{H}$. The base cycle of $X$ lies in a coset of a maximal compact subgroup of the group $\widehat{P} / \widehat{H}$ and a comparison of their dimension yields $d_{Y} \leq d_{X}$.

Remark 3.3. a) The assumption that the $G-$ orbit is open is needed. If $G$ is a real form in $\widehat{G}$, a complex semisimple Lie group, then $d_{\widehat{G}}>d_{G}$.

b) The semisimple assumption is also needed. In the classification of homogeneous complex surfaces OR84 the example

$$
Y=\widehat{G} / \widehat{H} \cong \mathbb{C}^{2} \supset X=G / H=\mathbb{C}^{2} \backslash \mathbb{R}^{2}
$$

arises in two ways. One way is with solvable groups, see Proposition 5.2 in loc. cit. The other way which is relevant to our present considerations is via the real form $G=S L_{2}(\mathbb{R}) \ltimes \mathbb{R}^{2}$ of the mixed group $\widehat{G}=S L_{2}(\mathbb{C}) \ltimes \mathbb{C}^{2}$, see Theorem 6.3 in loc. cit. Here $d_{Y}=\operatorname{dim}_{\mathbb{R}} Y=4>3=d_{X}$, since $X$ is topologically $\mathbb{S}^{1} \times \mathbb{R}^{3}$.

\section{Proof of the Theorem}

With these preparations we can now give the proof of the Theorem.

Proof. Let $G / H \rightarrow G / J$ be the $\mathfrak{g}$-anticanonical fibration, e.g., see HO84] and OR84. If $J=G$, then $G$ has the structure of a complex Lie group and is acting holomorphically on $X$ and thus $X$ already has the desired form, e.g., see Corollary 4, p. 64 in $\mathrm{HO} 84$. Note that $H$ is discrete in this case.

So we assume that $G \neq J$. Then $G / J$ and $J / H$ are complex manifolds and it follows from the Fibration Lemma AG94 that $d_{G / J} \leq 2$. Further, the bundle $G / H^{\circ} \rightarrow G / J$ is a locally trivial holomorphic principal fiber bundle with structure group the complex Lie group $J / H^{\circ}$, where $H^{\circ}$ denotes the connected component of the identity of the group $H$. Moreover, the base $G / J$ of the $\mathfrak{g}-$ anticanonical fibration is a $G$-orbit in some $\mathbb{P}_{N}$ and is open in the corresponding $\widehat{G}-$ orbit $Y:=\widehat{G} / \widehat{J}$, where $\widehat{G}$ denotes the smallest connected complex subgroup of the automorphism group $\operatorname{Aut}\left(\mathbb{P}_{N}\right)$ containing $G$.

If we show that the $G$-action on the base $G / J$ can be extended to a $\widehat{G}$-action on $G / J$, then we can extend the $G$-action on $\tilde{X}:=G / H^{\circ}$ to a $\widehat{G}$-action on $\tilde{X}$ (Proposition 2.6, GH98]) and this then defines a $\widehat{G}$-action on $X$. Note that if $d_{G / J}=0$, then $G / J=\widehat{G} / \widehat{J}$ is compact and thus is a flag manifold and we have the desired extension. And if $d_{G / J}=1$, then the result follows by Theorem 3.1 in GH98. Hence we assume that $d_{G / J}=2$ throughout the rest of the proof.

Now, as a consequence of a Theorem of Chevalley Che51, the commutator subgroup $(\widehat{G})^{\prime}$ of $\widehat{G}$ has closed orbits in $Y$ and this yields the following fibrations

$$
\begin{array}{ccc}
G / J & \hookrightarrow & \widehat{G} / \widehat{J} \\
\downarrow & & \downarrow \\
G / I & \hookrightarrow & \widehat{G} / \widehat{J} \cdot(\widehat{G})^{\prime}
\end{array}
$$

where $I:=G \cap / \widehat{J} \cdot(\widehat{G})^{\prime}$. The base $\widehat{G} / \widehat{J} \cdot(\widehat{G})^{\prime}$ is a Stein Abelian Lie group; see the Lemma on p.173 in [HO81. Since $G / I$ is open in the connected Abelian Lie group 
$\widehat{G} / \widehat{J} \cdot(\widehat{G})^{\prime}$, it follows that $G / I=\widehat{G} / \widehat{J} \cdot(\widehat{G})^{\prime}$. If $\operatorname{dim} G / I>0$, then $d_{G / I}>0$, since $G / I$ is Stein. Also $2=d_{G / J} \geq d_{I / J}+d_{G / I}$ by the Fibration Lemma in [AG94]. If $d_{G / I}=2$, then $I / J$ is compact and $X=Y$, so we are done. And if $d_{G / I}=1$, then $d_{I / J}=1$. Then the complexification of $I$ acts transitively on $I / J$ by Theorem 3.1 in GH98, and this implies $\widehat{G}$ acts transitively on $X$. Again we are done.

So we may assume that $I=G$ and $\left(\widehat{G)^{\prime}}\right.$ is acting transitively and as an algebraic group on $Y$. Then the orbits of its radical $R_{(\widehat{G})^{\prime}}$ are closed and these orbits give rise to the following fibrations

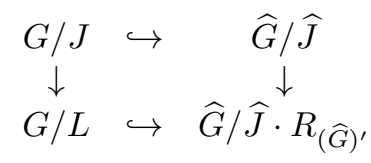

where $L:=G \cap \widehat{J} \cdot R_{(\widehat{G})^{\prime}}$. If $\operatorname{dim} L / J>0$, then we apply Lemma 5 in Gil95] where it is shown that $d_{L / J}=2$ and either a complex Lie group acts transitively on $G / J$ or else $G / J$ fibers as a unit disk bundle over the compact base $G / L$ thus proving the result in this setting.

Otherwise, $R_{(\widehat{G})^{\prime}}$ is acting ineffectively and we may assume that $G$ and $\widehat{G}$ are both semisimple. Now let $M$ be the connected subgroup of $G$ corresponding to the maximal complex ideal $\mathfrak{m}:=\mathfrak{g} \cap i \mathfrak{g}$ in the Lie algebra $\mathfrak{g}$. The group $M$ is algebraic and has closed orbits in $Y$ and the fibers of the fibrations of $X$ and $Y$ induced by these $M$ orbits are equal. Hence we may assume that $\mathfrak{m}=(0)$ and thus the problem reduces to the setting where $G$ is a real form of a connected complex semisimple Lie group $\widehat{G}$.

Now Lemma 3.1 applies and $d_{Y} \leq d_{X}=2$. So $d_{Y}=0,1$, or 2 .

Suppose first that $d_{Y}=0$ and so $Y$ is compact. Then $X=G / J$ is a flag domain in the flag manifold $Y=\widehat{G} / \widehat{J}$, where $G$ is a real form of a semisimple complex Lie group $\widehat{G}$. Then $Y$ splits into a product of flag manifolds of the simple factors of $\widehat{G}$ (modulo ineffectivity). The flag domain $X$ splits into a corresponding product of flag domains in each flag manifold, see (p 1147 in Wol69]). Now we can ignore factors $X_{i}$ that are compact (the complexification of the simple real form acts transitively on such factors) and that satisfy $d_{X_{i}}=1$ (again the complexification acts transitively by Theorem 3.1 in GH98]). Thus we are reduced to consideration of one factor having $d=2$ and all other factors compact. Such a flag domain of a real form of a complex semisimple Lie group is handled by Proposition 2.1

Next assume that $d_{Y}=1$. By the result in [Akh77, we get the following fibrations

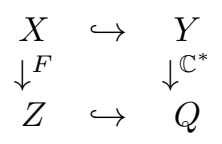

where $Q$ is a flag manifold. Since $F$ is an open orbit in $\mathbb{C}^{*}$, we have $F=\mathbb{C}^{*}$. Hence $d_{Z}=1$, and $\widehat{G}$ acts on $Z$ (Theorem 3.1, GH98) and thus there is a transitive $\widehat{G}$ action on $X$. We are again done by our observations above.

Finally we consider the case $d_{Y}=2$. We are in a setting where a linear algebraic group is acting on $Y$ and Akhiezer Akh83 proved that the complex manifold $Y$ 
has the following fibration which induces a corresponding fibration of $X$ :

$$
\begin{array}{ccc}
X & \hookrightarrow & Y \\
\downarrow F & & \downarrow \widehat{F} \\
G / U & \hookrightarrow & \widehat{G} / \widehat{P}
\end{array}
$$

where $\widehat{P}$ is a parabolic subgroup of $\widehat{G}, U:=\widehat{P} \cap G$ and $\widehat{F}$ is $\mathbb{C}, \mathbb{C}^{*} \times \mathbb{C}^{*}$, or $\mathbb{P}_{2}$ minus a quadric curve. Note that since $F$ is open in the Stein space $\widehat{F}$, one has $d_{F} \neq 0$. If $d_{F}=1$, then $F=\mathbb{C}^{*}$ and this case does not occur. Hence the remaining case is when $d_{F}=2$. If the manifold $\widehat{F}$ is biholomorphic to $\mathbb{C}^{*} \times \mathbb{C}^{*}$, or $\mathbb{P}^{2} \backslash Q$ with $Q$ a quadric curve, then the transitive action of $\widehat{P}$ on $\widehat{F}$ is given by group multiplication in the first case and the action of $\operatorname{PSL}(2, \mathbb{C})$ by projective transformations in the second. In both cases there is no Lie subgroup with an open orbit $F$ having $d_{F}=2$. Otherwise, $\widehat{F}=\mathbb{C}$ with the complex two dimensional affine group acting transitively, see Akh83, and $F$ can then be the upper half plane as the orbit of the real affine group. Once again the unit disk occurs as the fiber of a bundle over a flag manifold and this completes the proof of the Theorem.

\section{REFERENCES}

[Abe76] H. Abels, Proper transformation groups. Transformation groups. (Proc. Conf., Univ. Newcastle upon Tyne, Newcastle upon Tyne, 1976), pp. 237 - 248. London Math. Soc. Lecture Note Series, No. 26, Cambridge Univ. Press, Cambridge, 1977.

[Abe82] H. Abels, Some topological aspects of proper group actions; noncompact dimension of groups. J. London Math. Soc. (2) 25 (1982), no. 3, 525 - 538.

[Akh77] D. N. Ahiezer, Dense orbits with two endpoints. (Russian) Izv. Akad. Nauk SSSR Ser. Mat. 41 (1977), no. 2, 308 - 324; Engl. transl.: Math. USSR-Izv. 11 (1977), no. 2, 293 - 307 (1978).

[Akh79] D. N. Ahiezer, Algebraic groups that are transitive in the complement to a homogeneous hypersurface, (Russian) Dokl. Akad. Nauk SSSR 245 (1979), no. 2, 281 - 284; Engl. transl.: Soviet Math. Dokl. 20 (1979), no. 2, $278-281$.

[Akh83] D. N. Akhiezer, Complex n-dimensional homogeneous spaces homotopically equivalent to $(2 n-2)$-dimensional compact manifolds. Selecta Math. Sov. 3, no.3 (1983/84), $286-290$.

[Akh13] D. N. Akhiezer, Real group orbits on flag manifolds, in Lie Groups: Structure, Actions, and Representations, In Honor of Joseph A. Wolf on the Occasion of his 75th Birthday, pp 1 - 24, Progress in Mathematics 306, Birkhäuser, 2013.

[AG94] D. Akhiezer and B. Gilligan, On complex homogeneous spaces with top homology in codimension two. Canad. J. Math. 46 (1994), 897 - 919.

[BoMo47] S. Bochner and D. Montgomery, Groups on analytic manifolds. Ann. of Math. 48 (1947), $659-669$.

[Bor53] A. Borel, Les bouts des espaces homogènes de groupes de Lie. Ann. of Math. (2) 58 (1953), $443-457$.

[Che51] C. Chevalley, Théorie des Groupes de Lie. Tome II. Groupes Algébriques. Hermann \& Cie., Paris, 1951.

[FHW] G. Fels, A. T. Huckleberry, and J. Wolf, Cycle spaces of flag domains. A complex geometric viewpoint. Progress in Mathematics, 245 Birkhäuser Boston, Inc., Boston, MA, 2006.

[Gi191] B. Gilligan, On the ends of complex manifolds homogeneous under a Lie group. Proc. Sympos. Pure Math., 52, Part 2, Amer. Math. Soc., Providence, RI, 1991.

[Gi195] B. Gilligan, Complex homogeneous spaces of real groups with top homology in codimension two. Ann. Global Anal. Geom. 13 (1995), 303 - 314.

[GH98] B. Gilligan and P. Heinzner, Globalization of holomorphic actions on principal bundles. Math. Nachr. 189 (1998), $145-156$.

[GH09] B. Gilligan and A. T. Huckleberry, Fibrations and globalizations of compact homogeneous CR-Manifolds. (Russian. Russian summary) Izv. Ross. Akad. Nauk Ser. Mat. 73 (2009), no. 3, 67 - 126; Engl. transl.: Izv. Math. 73 (2009), no. 3, $501-553$. 
[Hir70] H. Hironaka, Desingularizations of complex-analytic varieties. Actes Congrès intern. Math. 2 (1970). $627-631$.

[Hoch65] G. Hochschild, The Structure of Lie Groups. Holden-Day Inc., San Franscisco-LondonAmsterdam, 1965.

[HO81] A. T. Huckleberry and E. Oeljeklaus, Homogeneous Spaces from a Complex Analytic Viewpoint. Manifolds and Lie groups. (Notre Dame, Ind., 1980) p,. 159 - 186, Progress in Math., 14 Birkhäuser, Boston (1981).

[HO84] A. T. Huckleberry and E. Oeljeklaus, Classification theorems for almost homogeneous spaces. Institut Élie Cartan, 9. Université de Nancy, Institut Élie Cartan, Nancy, 1984.

[HS81] A. T. Huckleberry and D. Snow, A classification of strictly pseudoconcave homogeneous manifolds. Ann. Scuola Norm. Sup. Pisa 8 (1981), $231-255$.

[HS82] A. T. Huckleberry and D. Snow, Almost-homogeneous Kähler manifolds with hypersurface orbits, Osaka J. Math. 19 (1982), 763 - 786.

[Ka67] W. Kaup, Reelle Transformationsgruppen und invariante Metriken auf komplexen Räumen, Invent. Math. 3 (1967), 43 - 70.

[Mal75] F. M. Malyshev, Complex homogeneous spaces of semisimple Lie groups of the first category, Izv. Akad. Nauk SSSR, ser. mat. 39, 5 (1975), 992 - 1002, Engl. transl.: Math. USSR Izv. 9, 5 (1977), 939 - 949.

[Mal77] F. M. Malyshev, Complex homogeneous spaces of semisimple Lie groups of type $D_{n}$, Izv. Akad. Nauk SSSR, ser. mat. 41, 4, (1977), 829 - 852, Engl. transl.: Math. USSR Izv. 11, 4 (1977), $783-805$.

[Mat79] T. Matsuki, The orbits of affine symmetric spaces under the action of minimal parabolic subgroups. J. Math. Soc. Japan 31 (1979), no. 2, 331 - 357.

[Mat82] T. Matsuki, Orbits on affine symmetric spaces under the action of parabolic subgroups. Hiroshima Math. J. 12 (1982), 307 - 320.

[Mat60] Y. Matsushima, Espaces homogènes de Stein des groupes de Lie complexes, Nagoya Math. J. 16 (1960), $205-218$.

[MN63] A. Morimoto and T. Nagano, On pseudo-conformal transformations of hypersurfaces. J. Math. Soc. Japan. 15 No. 3, 1963, 289 - 300.

[Mos55] G. D. Mostow, On covariant fiberings of Klein spaces. Amer. J. Math. 77 (1955). 247 278 and Covariant fiberings of Klein spaces. II. Amer. J. Math. 84 (1962), $466-474$.

[OR84] K. Oeljeklaus and W. Richthofer, Homogeneous complex surfaces. Math. Ann. 268 (1984), no. 3, $273-292$.

[Oni60] A.L. Onishchik, Complex hulls of compact homogeneous spaces, Dokl. Akad. Nauk SSSR 130726 - 729; English trans. Soviet Math. Dokl. 1 (1960) $88-91$.

[Oni62] A.L. Onishchik, Inclusion relations among transitive compact transformation groups, Trudy Mosk. Mat. Obshch. 11 (1962), 199 - 242, English transl.: Amer. Math. Soc. Transl. (2) $\mathbf{5 0}$ (1966), 5 - 58.

[Oni69] A.L. Onishchik, Decompositions of reductive Lie groups, Mat. Sbornik 80 (122) (1969), 553 - 599; Engl. transl.: Math. USSR Sb. 9 (1969), 515 - 554.

[Ste82] M. Steinsiek, Transformation groups on homogeneous-rational manifolds, Math. Ann. 260 (1982), $423-435$.

[Wan52] H. C. Wang, Two-point homogeneous spaces. Ann. of Math. (2) 55 (1952). 177 - 191.

[Weis66] Boris Weisfeiler, On one class of unipotent subgroups of semisimple algebraic groups, Uspehi Mat. Nauk 21 (1966), 222 - 223.

[Wol69] J. A. Wolf, The action of a real semisimple group on a complex flag manifold. I. Orbit structure and holomorphic arc components. Bull. Amer. Math. Soc. 75 (1969), $1121-1237$.

Department of Mathematics and Statistics, University of Regina, Regina, Canada S4S 0A2

E-mail address: seyed.ruhala.ahmadi@gmail.com and gilliganbc@gmail.com 